\title{
Acknowledgment to Reviewers of Risks in 2020
}

\section{Risks Editorial Office}

Citation: Risks Editorial Office. 2021. Acknowledgment to Reviewers of Risks in 2020. Risks 9: 28. https:// doi.org/10.3390/risks9020028

Published: 24 January 2021

Publisher's Note: MDPI stays neutral with regard to jurisdictional claims in published maps and institutional affiliations.

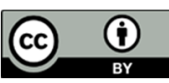

Copyright: (C) 2021 by the author. Licensee MDPI, Basel, Switzerland. This article is an open access article distributed under the terms and conditions of the Creative Commons Attribution (CC BY) license (http://creativecommons.org/licenses /by/4.0/).

MDPI AG, St. Alban-Anlage 66, 4052 Basel, Switzerland

Peer review is the driving force of journal development, and reviewers are gatekeepers who ensure that Risks maintains its standards for the high quality of its published papers. Thanks to the cooperation of our reviewers, in 2020, the median time to first decision was 18 days and the median time to publication was 45 days. The editors would like to express their sincere gratitude to the following reviewers for their precious time and dedication, regardless of whether the papers were finally published:

Abi Jaber, Eduardo

Abreu, António

Adamko, Peter

Adeyinka, Adewuyi Ayodele

Agliardi, Rossella

Agosto, Arianna

Aharon, David Yechiam

Akhundjanov, Sherzod

Alavi, Reza

Alfeus, Mesias

Anagnoste, Sorin

Andries, Alin

Anton, Sorin

Antuchevičienè, Jurgita

Antunes, Francisco

Arratia, Argimiro

Ashraf, Badar Nadeem

Aurell, Alexander

Avarmaa, Mari

Avram, Florin

Ayanou, Tilahun

Ayuso, Mercedes

Bace, Edward

Balbás, Alejandro

Baležentis, Tomas

Ballestra, Luca Vincenzo

Barbu, Viorel

Barbulescu, Alina

Barcik, Agnieszka

Barro, Diana

Barth, James R.

Beretta, Edoardo

Boado-Penas, María Del Carmen

Bogataj, David

Bohdalová, Mária
Boitan, Iustina Alina

Bolancé, Catalina

Borland, Lisa

Bostan, Ionel

Botlik, Josef

Bouri, Elie

Bragin, Mikhail

Brezeanu, Petre

Brooks, Robert

Brotons, Jose $\mathrm{M}$.

Busu, Cristian

Butnaru, Gina Ionela

Cachanosky, Nicolas

Cai, Jun

Čámská, Dagmar

Candila, Vincenzo

Cansado-Bravo, Pablo

Caraiani, Petre

Cary, Michael

Casteren, Jan Van

Cergo, Julio A.

Chang, Chia-Lin

Chang, Tzu-Pu

Chang, Victor

Chen, James Ming

Cheng, Po-Keng

Chevallier, Julien

Chiodo, Elio

Choi, Myoung Shik

Christophe, Sauvey

Clemente, Gian Paolo

Coenders, Germà

Constantinescu, Corina

Crépey, Stéphane

Crespo, Nuno 
Criste, Adina

Cristea, Mirela

Cui, Zhenyu

Czech, Katarzyna

Dassios, Angelos

Dassios, Ioannis

Dayanandan, Ajit

De Pablo Valenciano, Jaime

Debón, Ana

Delcea, Camelia

Depken, Craig

Devolder, Pierre

Di Persio, Luca

Dias, Gustavo Fruet

Díaz-Meneses, Gonzalo

Dinis, Maria Alzira Pimenta

Dionne, Georges

Doh, Taeyoung

Dornean, Adina

Drachal, Krzysztof

Dragisa, Stanujkic

Dragota, Victor

Druica, Elena

Durica, Marek

Dvorak, Jaroslav

Dwivedi, Ashutosh Dhar

Eisenberg, Julia

Ekanayake, Ekanayake M.

Elbardan, Hany

Elvira, Nica

Emery, Ashley

Fanelli, Viviana

Feng, Runhuan

Fera, Marcello

Fernández-Prados, Juan Sebastián

Ferreira, Paulo

Ferretti, Paola

Fetaji, Bekim

Filipe, José António

Fiori, Anna Maria

Floreani, Josanco

Foglia, Matteo

Fontana, Claudio

Franc-Dąbrowska, Justyna

Francois, Pascal

Franke, Ulrik

Frei, Christoph

Frey, Rüdiger

Furmańczyk, Konrad

Fusai, Gianluca

Gan, Guojun
Gardijan Kedžo, Margareta

Garsztka, Przemysław

Gerrard, Russell

Ghimire, Shankar

Giacometti, Rosella

Giudici, Paolo

Gkillas, Konstantinos

Gocheva-Ilieva, Snezhana Georgieva

Goecke, Oskar

Gomez, Hector

Gómez-Déniz, Emilio

González-Velasco, Carmen

Grabowska, Sandra

Gregova, Elena

Grigutis, Andrius

Gruszczyński, Marek

Guerreiro, Gracinda R.

Guharay, Sabyasachi

Guijarro, Francisco

Guo, Qiheng

Guyon, Julien

Haas, Armin

Hadaś-Dyduch, Monika

Haiss, Peter R.

Hajilee, Massomeh

Hämäläinen, Joonas

Hamori, Shigeyuki

Hannig, Jan

Harms, Philipp

Hassani, Hossein

Hategan, Camelia Daniela

Henry, Jérôme

Heo, Wookjae

Hernández-Perlines, Felipe

Hernes, Marcin

Herzog, Bodo

Hieber, Peter

Hildmann, Hanno

Hodžić, Sabina

Huang, Chung-Jen

Huang, Kershen

Huang, Xin

Hung, Jui-Cheng

Huynh, Toan Luu Duc

Ianole-Calin, Rodica

Ishii, Hokuto

Iwanicz-Drozdowska, Małgorzata

Jang, Hyun Jin

Jędrzychowska, Anna

Jevtic, Petar

Ji, Hyun Mi 
Jiang, Wenjun

Jin, Zhuo

Jindrichovska, Irena

Johannssen, Arne

Jona, Gyorgy

Jones, Roger

Jureviciene, Daiva

Kaabia, Olfa

Kadry, Seifedine

Karbowski, Adam

Karlis, Dimitris

Katsikas, Sokratis

Kaucic, Massimiliano

Kaushansky, Vadim

Kebiri, Omar

Kenett, Dror

Kenett, Ron S.

Kim, Jinsoo

Klapkiv, Lyubov

Klein, Tony

Kliestik, Tomas

Koike, Yuta

Kolev, Mikhail

Kolkiewicz, Adam W.

Konstantinides, Dimitrios

Korbel, Jan

Korzeb, Zbigniew

Kovács, Sándor

Kowalski, Arkadiusz M.

Kratsios, Anastasis

Krištofík, Peter

Kumar, Kuldeep

Lars, Kirkby Justin

Lavagnini, Silvia

Leccadito, Arturo

Lee, Gee

Lee, Hee Soo

Lee, Jeong Hwan

Lee, Namryoung

Lelong, Jérôme

León, Angel

Li, Jackie

Li, Weiping

Liczmańska-Kopcewicz, Katarzyna

Lin, Xiang

Lio, Yuhlong

Lisa, Antonella

Liu, Fangda

Liu, Qingfeng

López, Bernardino Benito

López-Gutiérrez, Carlos
López-Pascual, Joaquín

Lotto, Josephat Daniel

Low, Rand

Lu, Su-Lien

Lyubov, Klapkiv

Maier-Paape, Stanislaus

Mailhot, Mélina

Maitah, Mansoor

Majerova, Jana

Makov, Udi

Malekian, Arash

Manta, Otilia

Marazzina, Daniele

Marcu, Nicu

Marfatia, Hardik A.

Marszk, Adam

Martin Cervantes, Pedro A.

Martínez, Francisco Guijarro

Martino, Luca

Mateus, Cesario

Matheson, Victor

Maury, Tristan-Pierre

Maza, Adolfo

Mazzù, Sebastiano

Mensah, Emmanuel Kwasi

Menzietti, Massimiliano

Mezei, József

Michaelides, Michael

Michalski, Grzegorz

Miglo, Anton

Milkau, Udo

Miller, Gregory

Miller, Stephen Matteo

Molodtsova, Tanya

Monge, Manuel

Montllor, Joan

Mora-Garcia, Raul-Tomas

Morkunas, Mangirdas

Morley, Bruce

Moskalev, Alexey

Mougoué, Mbodja

Moutanabbir, Khouzeima

Mullat, Joseph E.

Muroi, Yoshifumi

Naik, Punith

Nakagawa, Kei

Natalia, Aizenberg

Necula, Ciprian

Newall, Philip W. S.

Newaz, Mohammad

Newton, Andy 
Ng'ombe, John Nedson

Nguyen, Thi Thanh-Binh

Nicolau, Cristina

Nin, Jordi

Nuta, Alina Cristina

Oliva, Immacolata

Oosterlee, Cornelis W.

Orlando, Albina

Orlova, Ekaterina

Ortiz-Latorre, Salvador

Osipenko, Maria

Overill, Richard

Pablo-Martí, Federico

Pantelous, Athanasios

Paparas, Dimitrios

Paraskevopoulos, Ioannis

Park, Jaehwan

Pascariu, Marius D.

Pau, Giovanni

Paweł, Dymora

Pele, Daniel

Pellegrino, Roberta

Pelletier, Denis

Perote, Javier

Pešta, Michal

Picech, Liviana

Pipień, Mateusz

Pires Manso, José Ramos

Pirra, Marco

Piscopo, Gabriella

Plonis, Darius

Podhorská, Ivana

Popescu, Cristina Raluca Gh.

Porrini, Donatella

Porzio, Claudio

Powell, Robert J.

Preda, Vasile

Prömel, David

Ptak-Chmielewska, Aneta

Radanliev, Petar

Rakhmangulov, Aleksandr

Rambaud, Salvador Cruz

Ramsey, Austin Ford

Ranftl, Sascha

Rathie, Pushpa

Rathore, Heena

Raźniak, Piotr

Regis, Luca

Regoliosi, Carlo

Reimers, Hans-Eggert

Requier-Desjardins, Denis
Resta, Marina

Rjoub, Husam

Rogova, Elena

Rolf, Poulsen

Romaniuk, Maciej

Romanova, Inna

Rotondi, Francesco

Rupšys, Petras

Rutecka-Góra, Joanna

Safa, M. Faisal

Salamanca, Juan Jesús

Salhi, Yahia

Sarantsev, Andrey

Sarath, Bharat

Schlögl, Erik

Schlütter, Sebastian

Schneider, Jan

Senga Kiesse, Tristan

Sevic, Aleksandar

Shemyakin, Arkady

Shen, Xuan (Shelly)

Shen, Yang

Shushi, Tomer

Shust, Efrat

Siaulys, Jonas

Siddiqi, Hammad

Simões, Nádia

Simões, Paula

Sinha, Amit K.

Škrinjarić, Tihana

Ślusarczyk, Beata

Snudden, Stephen

So, Jacky Yuk-Chow

Sojli, Elvira

Sonenshine, Ralph

Sprincean, Nicu

Stankevičienè, Jelena

Stanuszek, Marek

Stark, Dudley

Stasiak, Michal

Strascia, Stefano Cavastracci

Strielkowski, Wadim

$\mathrm{Su}$, Jianxi

$\mathrm{Su}, \mathrm{Ye}$

Šutienè, Kristina

Swishchuk, Anatoliy

Szczepankiewicz, Elżbieta

Szpruch, Lukasz

Tang, Wanwei

Targino, Rodrigo

Tavin, Bertrand 
Tian, Ruilin

Tian, Weidong

Tichý, Tomáš

Tiganoaia, Bogdan

Tomášková, Hana

Tomczak, Sebastian

Toporowski, Jan

Trapin, Luca

Trinidad Segovia, Juan E.

Tsai, Feng-Tse

Tsionas, Mike

Tzougas, George

Uchida, Satoshi

Valdez, Emiliano A.

Valverde, Raul

Varela-Candamio, Laura

Vergos, Konstantinos

Vespri, Vincenzo

Veuger, Jan

Villegas, Andres

Vives, Josep

Vogiazas, Sofoklis

Vrbka, Jaromír

Vyrostova, Eva

Wang, Hai

Wang, Shen-Tsu

Wang, Yi-Hsien
Wichitaksorn, Nuttanan

Wieczorek-Kosmala, Monika

Wirjanto, Tony

Witkovsky, Viktor

Witzany, Jiří

Wójcicka-Wójtowicz, Aleksandra

Wong, Wing-Keung

$\mathrm{Wu}$, Kai

$\mathrm{Xu}$, Mengyi

$\mathrm{Xu}$, Xiaoqing Eleanor

Yam, Sheung

Yamazaki, Kazutoshi

Yang, Hailiang

Yang, Yipeng

Yavuz, Mehmet

Yoon, SungMan

Young, Derek

$\mathrm{Yu}$, Daoping

Zavadskas, Edmundas Kazimieras

Zhang, Jinhui

Zhao, Yichuan

Zhu, Lingjiong

Zhu, Sheng

Zhuang, Shengchao

Zia, Kashif

Zitikis, Ricardas

Zou, Bin 